\title{
Electrochemical Characterization of Streptavidin-HRP Immobilized on Multiwall Carbon Nanotubes for Biosensor Applications
}

\author{
Imene Hafaiedh, Hamdi Baccar, Taha Ktari, Adnane Abdelghani* \\ Nanotechnology Laboratory, National Institute of Applied Science and Technology, Tunis, Tunisia. \\ Email: *aabdelghan@yahoo.fr
}

Received October $17^{\text {th }}, 2011$; revised November $24^{\text {th }}, 2011$; accepted December $6^{\text {th }}, 2011$

\begin{abstract}
$\mathrm{n}$ this work, we used gold labeled multiwall carbons nanotubes for peroxidase biosensor. The gold labeling on multiwall carbon nanotubes can be achieved with Pressure vapor Deposition (PVD) technique. The obtained carbon nanotubes can be immobilized on gold electrode with the airbrushing technique. The stability and the molecular structure of the labeled multiwall carbon nanotubes were characterized with cyclic voltammetry, impedance spectroscopy and Fourrier Transform Infra-Red spectroscopy (FTIR). It shows a higher conductivity and a good stability in water interface. For streptavidin-HRP immobilization, the labeled gold nanotubes were activated over night with thiol-acid (16 carbons). An activation procedure was achieved with EDC/NHS for HRP-streptavidin immobilization. The development of biosensor for $\mathrm{H}_{2} \mathrm{O}_{2}$ detection was observed with the impedance spectroscopy and cyclic voltammetry techniques. This method could be used to determine total $\mathrm{H}_{2} \mathrm{O}_{2}$ concentration in the range $4 \mu \mathrm{M}-160 \mu \mathrm{M}$. The results show that the biosensor response depends on the conductivity and the large surface-to-volume ratio attained with multiwall carbon nanotubes. The response of the developed biosensors was reproducible with higher stability.
\end{abstract}

Keywords: Carbon Nanotubes; Biosensor; Impedance Spectroscopy; Cyclic Voltammetry

\section{Introduction}

Carbon nanotubes (CNT) have become the subject of intense investigation since their discovery [1]. Such considerable interest reflects the unique behavior of CNT, including their remarkable electrical, chemical, mechanical and structural properties. CNT can display metallic, semiconducting and superconducting electron transport process [2]. CNT can be made by chemical vapor deposition, carbon arc methods, or laser evaporation and can be divided into single wall carbon-nanotubes (SWCNT) and multi-wall carbon nanotubes (MWCNT). SWCNT possess a cylindrical nanostructure (with a high aspect ratio), formed by rolling up a single graphite sheet into a tube [3]. MWCNT comprise of several layers of graphene cylinders that are concentrically nested like rings of a tree trunk (with an interlayer spacing of $3.4 \AA$ ) [2,3]. The unique properties of carbon nanotubes make them extremely attractive for the task of chemical sensors, in general and electrochemical detection, in particular [3]. CNTmodified electrodes have been shown useful to accumulate important biomolecules (DNA, antibody) [3] to increase the surface/volume ratio. As an extension of the u-

\footnotetext{
"Corresponding author.
}

sual functionalized multiwall carbon nanotubes $(-\mathrm{COOH}$, $-\mathrm{NH}_{2}$ ), they can be decorated with metal na- noparticles. Since metal clusters show a wide range of advanced physic-chemical properties (e.g. high catalytic activity, adsorption capacity, efficient charge transfer, etc. ‥) [4]. In this work, we used gold labeled multiwall carbon nanotubes for peroxidase biosensor. The multiwall carbon nanotubes can be immobilized on gold electrode with the airbrushing technique. The stability and the molecular structure of the metal labeled multiwall carbon nanotube were characterized with cyclic voltammetry, impedance spectroscopy and Fourrier Transform Infra-Red spectroscopy (FTIR). It shows a higher conductivity and a good stability in water interface. For streptavidin-HRP immobilization, the labeled gold nanotubes were activated over night with thiol-acid (16 carbons). An activation procedure was achieved with EDC/NHS for HRP-streptavidin immobilization. The development of biosensor for the enzymatic determination of $\mathrm{H}_{2} \mathrm{O}_{2}$ was observed with the impedance spectroscopy and cyclic voltammetry techniques. This method could be used to determine total $\mathrm{H}_{2} \mathrm{O}_{2}$ concentration in the range $4 \mu \mathrm{M}-160 \mu \mathrm{M}$. The response of the developed biosensors was reproducible with higher stability. 


\section{Materials and Methods}

\subsection{Reagents}

All other materials, including thiol-acid (16-mercaptohexadecanoic acid) (Sigma-Aldrich), 1-ethyl-3-(3-(dimethylamino)-propyl)carbodiimide (EDC) (Aldrich), and Nhydroxy succinimide (NHS) (Aldrich) were used as supplied. The gold labeling MWCNTs (Nanocyl, Belgium) were grown by PVD (Pressure Vapor Deposition) with purity higher than $95 \%$. Nanotubes were up to $50 \mathrm{mi}$ crometer in length and their outer and inner diameters ranged from $3 \mathrm{~nm}$ to $15 \mathrm{~nm}$ and $3 \mathrm{~nm}$ to $7 \mathrm{~nm}$, respectively.

The buffer solution used for all experiments was phosphate buffered saline (PBS) containing $140 \mathrm{mM} \mathrm{NaCl}$, $2.7 \mathrm{mM} \mathrm{KCl}, 0.1 \mathrm{mM} \mathrm{Na}_{2} \mathrm{HPO}_{4}, 1.8 \mathrm{mM} \mathrm{KH}_{2} \mathrm{PO}_{4}, \mathrm{pH}=$ 7 and the redox couple $\mathrm{Fe}(\mathrm{CN})_{6}^{3-} / \mathrm{Fe}(\mathrm{CN})_{6}^{4-}$ at a $5 \mathrm{mM}$ concentration. All reagents were of analytical grade and ultrapure water (resistance $18.2 \mathrm{M} \Omega \cdot \mathrm{cm}^{-1}$ ) produced by a MilliporeMilli-Q system was used.

\subsection{Gold Cleaning and Functionalization}

The gold electrodes $(1 \mathrm{~cm} \times 1 \mathrm{~cm})$ fabricated at the National Center of Microelectronics of Barcelona (Spain). Evaporated gold ( $300 \mathrm{~nm}$ thickness) was deposited on silicon, using a titanium underlayer ( $\sim 30 \mathrm{~nm}$ thickness) as substrate. Before modification, the gold electrodes were cleaned in acetone solution for $10 \mathrm{~min}$ with ultrasonic bath. After that, they were dried under a nitrogen flow and then dipped for $10 \mathrm{~min}$ into "piranha solution" 7:3 (v/v) $96 \% \mathrm{H}_{2} \mathrm{SO}_{4} / 30 \% \mathrm{H}_{2} \mathrm{O}_{2}$. Finally, the gold substrates were rinsed 2 to 3 times with ultra-pure water and immediately immersed in an ethanol solution. After cleaning, the gold labeled multiwall carbon nanotubes was dissolved in acetone and deposited on gold electrode with the Airbrushing technique at $90^{\circ} \mathrm{C} \mathrm{[5].} \mathrm{Immediately} \mathrm{after,} \mathrm{the}$ substrate was treated for two hours at $120^{\circ} \mathrm{C}$. More details concerning the synthesis and deposition of the gold labeled multiwall carbon nanotubes can be found in reference [6].

After, the gold electrode was immersed in a solution containing $1 \mathrm{mM}$ of Carboxyl thiol (16 carbons) in PBS buffer for 12 hours at room temperature. The gold labeling the outer surface of the carbon nanotubes will react specifically with the thiol. The treated electrode was then immersed in a solution of EDC $(0.4 \mathrm{mM})$ and NHS (0.1 $\mathrm{mM}$ ) for $1 \mathrm{~h}$. A $10 \mu \mathrm{l}$ of HRP solution with a concentration of $100 \mathrm{~b} \mu \mathrm{g} / \mathrm{ml}$ was deposited on the treated electrode for 1 hour. Then, a $10 \mu \mathrm{l}$ of $1 \%$ BSA solution was added on the substrate for 30 min to block the free spaces between the enzyme and the thiol.

\subsection{Electrochemical Set-Up}

\subsubsection{Cyclic Voltammetry}

Cyclic voltammetry was performed at room temperature in a conventional voltammetric cell with a three electrode configuration using Autolab impedance anlayzer (Ecochemie, Netherland). The gold electrode $\left(0.16 \mathrm{~cm}^{2}\right)$ was used as working electrode, platinum $\left(1 \mathrm{~cm}^{2}\right)$ and $\mathrm{Ag} / \mathrm{Ag}$ $\mathrm{Cl}$ electrodes were used as counter and reference electrodes respectively. All the electrochemical measurement were carried out in PBS at $\mathrm{pH} 7.0$ with $5 \mathrm{mM} \mathrm{Fe}(\mathrm{CN})_{6}^{3-}$ / $\mathrm{Fe}(\mathrm{CN})_{6}^{4-}$ and in Faraday cage.

\subsubsection{Impedance Spectroscopy}

The impedance analysis was performed with the Autolab $302 \mathrm{~N}$ impedance analyzer (Eco-Chemie, Netherland) in the frequency range $0.05 \mathrm{~Hz}-100 \mathrm{kHz}$, using a modulation voltage of $10 \mathrm{mV}$. More details on electrochemical impedance spectroscopy can be found in reference [7$15]$.

\subsubsection{Fourier Transform Infra-Red Spectroscopy}

To confirm the immobilization of metal labeled multiwall carbon nanotubes on gold surface, we used an ATRFTIR spectrometer (BRUKER-IFS-66V/S) equipped with a Germanium $(\mathrm{Ge})$ monoreflection prism with a fixed incident angle of $45^{\circ}$ and a DTGS detector. All spectra were recorded at $4 \mathrm{~cm}^{-1}$ resolution with 128 scans, from $400 \mathrm{~cm}^{-1}$ to $4000 \mathrm{~cm}^{-1}$. Our method consisted on recording the spectrum of the cleaned gold substrate and then the gold substrate with MCWNTs film. The spectrum of the cleaned electrode served as a reference. The ratio of the two spectra gave the spectrum of the MCWNTs.

\section{Results and Discussions}

\subsection{Characterization of Gold Labeled Multiwall Carbon Nanotubes Layer}

\subsubsection{Cyclic Voltammetry}

Cyclic voltammetry is an electrochemical technique which can be used to study the kinetic of redox reactions of materials, their insulating and conducting properties. $\mathrm{Cy}$ clic voltammograms of the gold electrode (Figure 1(a)) shows a reversible wave which is the typical behavior of gold surface in the presence of the redox couple.

As is shown in Figure 1(a), the cyclic voltammogra$m$ defines the characteristics of a diffusion controlled redox process observed at the cleaned bare gold electrode. After modification of the gold surface with the gold labeled multiwall carbon nanotube layer, the current increase due to the high conductivity properties of the MWCNTs layer (Figure 1(b)). The success immobilization of multiwall carbon nanotube layer was confirmed with the current increase. 


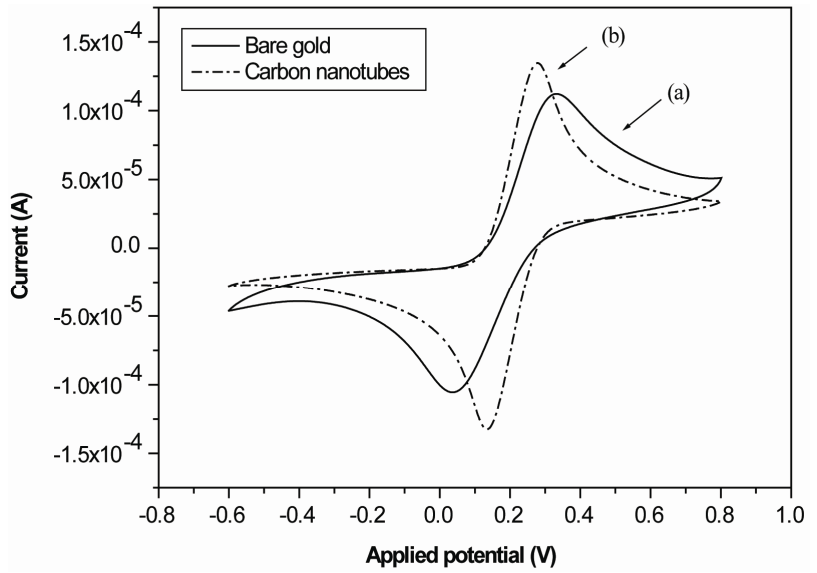

Figure 1. (a) Cyclic voltammograms of the gold electrode shows a reversible wave which is the typical behavior of goId surface in the presence of the redox couple; (b) Cyclic voltammograms of the gold electrode after MWCNTs deposition shows an increase of the current.

\subsubsection{Impedance Measurement}

Electrochemical Impedance Spectroscopy (EIS) is an effective tool for probing the feature surface-modified electrode while controlling its electrical properties [7-15]. This technique was used for example for the characterization of impedance behavior of SAM modified gold electrode [10-15]. Figure 2 shows Nyquist plots for gold electrode before and after MWCNTs deposition at $0 \mathrm{~V}$ with redox couple, where $\operatorname{Re}(z)$ is the real part and $\operatorname{Im}(z)$ is the imaginary part of the complex impedance $Z$.

The diameter of semi-circle corresponds to the charge transfer resistance of the electrode/electrolyte interface. The impedance spectra should be fitted using the electric model presented in Figure 3.

The capacitance $\mathrm{C}_{2}$ is the double layer capacitance of the gold/electrolyte interface, $R_{1}$ is the charge transfer resistance in low frequency range and $Z_{w}$ is the impedance due to the mass transfer of the redox species to the electrode described by Warburg. The resistance in high frequency ranges $\mathrm{R}_{0}$ is the resistance of the electrolyte, the contacts and connections.

The decrease of the charge transfer resistance after MWCNTs immobilization (Figure 2(b)) is due to the higher conductivity increases at the gold-electrolyte interface. This confirms the results obtained with cyclic voltammetry. The charge transfer resistance before and after MCWNTs deposition were extracted from the fitted data (Table 1).

\subsubsection{FTIR}

The infrared absorption spectra of gold electrode without and with MCWNTs are showed in Figure 4. Two main bands at $2900 \mathrm{~cm}^{-1}$ and $2984 \mathrm{~cm}^{-1}$ are clearly observed in the high frequency region.
These are assigned to the symmetric mode and the antisymmetric mode of $\mathrm{CH}_{2}$ respectively. The peaks located at $885 \mathrm{~cm}^{-1}, 1061 \mathrm{~cm}^{-1}, 1243 \mathrm{~cm}^{-1}$ and $1400 \mathrm{~cm}^{-1}$ should be assigned to $\mathrm{C}-\mathrm{H}$ out-of-plane deformation, $\mathrm{C}$ $\mathrm{H}$ out of plane ring deformation, $\mathrm{C}-\mathrm{C}$ out-of-plane ring deformation and $\mathrm{CH}_{2}$ scissor vibration mode respectively. This is confirming the deposition of MCWNTs on gold surface.

\subsection{Characterization of Self Assembled Monolayers (SAMs) Deposited on Gold Labeled Multiwall Carbon Nanotubes for HRP-Streptavidin Immobilization}

\subsubsection{Cyclic Voltammetry}

Figure 5 shows the cyclic voltammograms of the gold electrode with MWCNTs before (Figure 5(a)) and after thiol deposition (Figure 5(b)).

The current decreases due to the high insulating properties of thiol layer. This is confirming the success deposition of thiol on gold labeling MWCNTs. The same behavior was obtained after streptavidin-HRP immobilization (Figure 5(c)).

\subsubsection{Impedance Spectroscopy}

Figure 6 shows the Nyquist impedance plots for gold electrode with MWCNTs before (Figure 6(a)) and after thiol deposition (Figure 6(b)) at $0 \mathrm{~V}$.

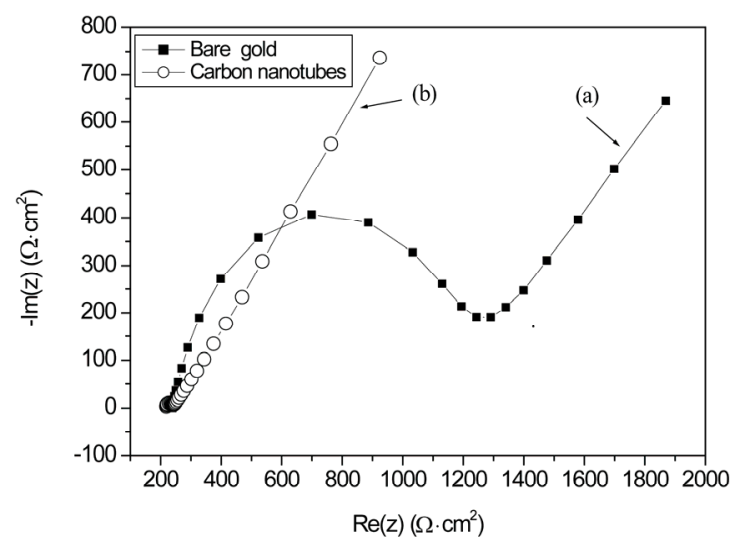

Figure 2. (a) Nyquist impedance plots for gold electrode with redox couple at $0 \mathrm{~V}$; (b) Nyquist impedance plots for gold electrode after MWCNTs deposition with redox couple at $0 \mathrm{~V}$.

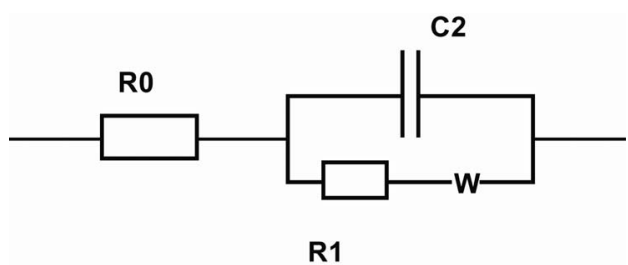

Figure 3. Electric model. 
Table 1. The charge transfer resistance extracted from the fitted data.

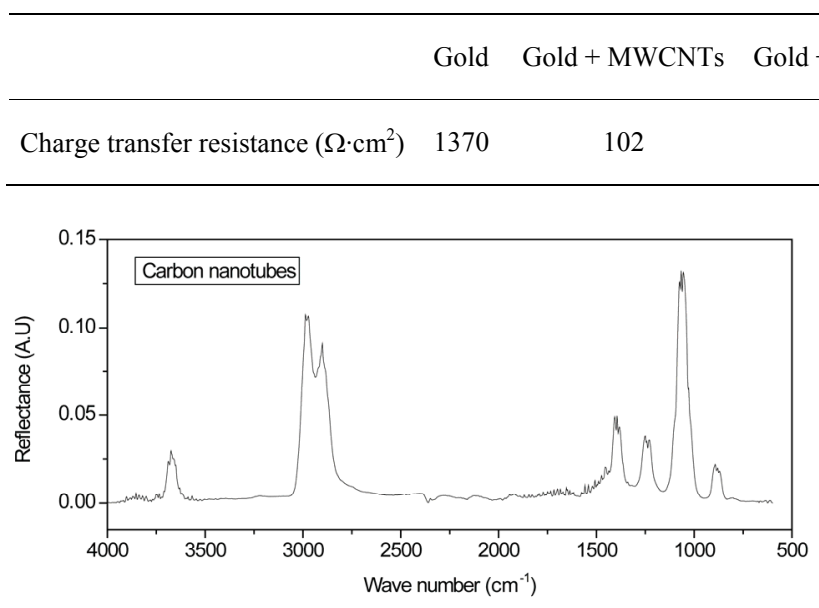

Figure 4. Infrared absorption spectra of gold electrode with MCWNTs.

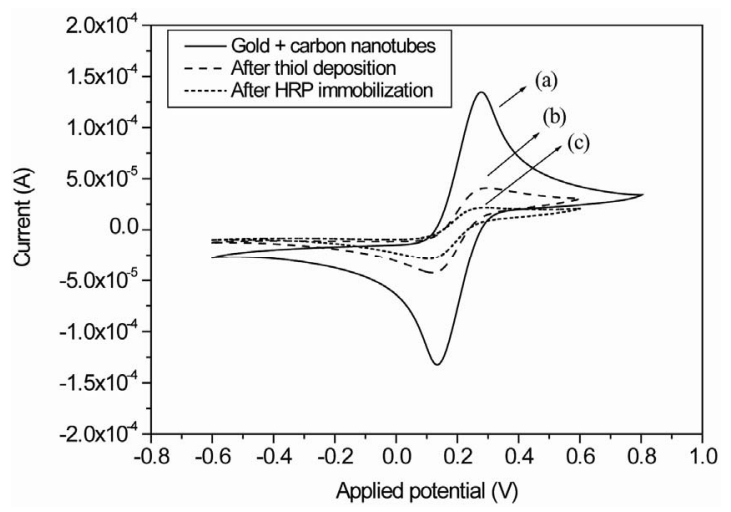

Figure 5. (a) Cyclic voltammograms of the gold electrode with MWCNTs; (b) Cyclic voltammograms of the gold electrode with MWCNTs after thiol deposition; (c) Cyclic voltammograms of the gold electrode with MWCNTs treated with thiol and after Streptavidin-HRP immobilization.

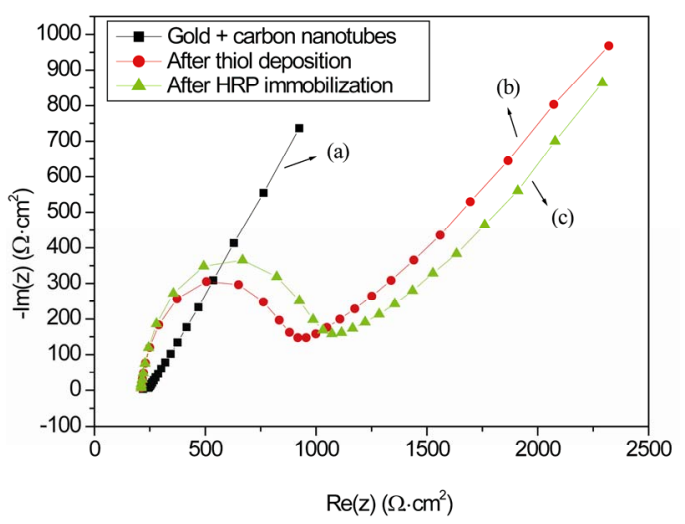

Figure 6. (a) Nyquist impedance plots for gold electrode with MWCNTs at $0 \mathrm{~V}$; (b) Nyquist impedance plots for gold electrode with MWCNTs after thiol at $0 \mathrm{~V}$; (c) Nyquist impedance plots for gold electrode with MWCNTs treated with thiol after streptavidin-HRP immobilization at $0 \mathrm{~V}$.
The semi-circle diameter which corresponds to the charge transfer resistance of the electrode/electrolyte interface was increasing. This is due to thiol deposition on gold labeling MWCNTs. The impedance spectra should be fitted using the electric model presented in Figure 3. We can estimate the percentage of covered area $(1-\theta)$ of the thiol, which can be described as:

$$
1-\theta=\frac{\mathrm{R}_{1} \text { before thiol deposition }}{\mathrm{R}_{1} \text { after thiol deposition }}
$$

We calculate the ratio and we obtain $(1-\theta)=90 \%$ which is a high coverage area.

The same behavior was obtained after strepatavidinHRP immobilization (Figure 6(c)). The charge transfer resistance before and after HRP deposition were extracted from fitted data (Table 1).

\subsection{Biosensor Application}

Figure 7 shows the cyclic voltammogram of the gold electrode functionalized with streptavidin-HRP enzyme before and after injection of $\mathrm{H}_{2} \mathrm{O}_{2}$.

Upon the addition of the hydrogen peroxide to the electrochemical cell, the reduction peak appears at $-200 \mathrm{mV}$ [16] showing a typical electron transfer between the $\mathrm{H}_{2} \mathrm{O}_{2}$ and the HRP molecule:

$$
\begin{aligned}
\mathrm{H}_{2} \mathrm{O}_{2}+\mathrm{HRP}_{\mathrm{red}} & \longrightarrow \mathrm{HRP}_{\mathrm{ox}}+\mathrm{H}_{2} \mathrm{O} \\
\mathrm{HRP}_{\mathrm{ox}}+2 \mathrm{e}^{-} & \longrightarrow \mathrm{HRP}_{\mathrm{red}}
\end{aligned}
$$

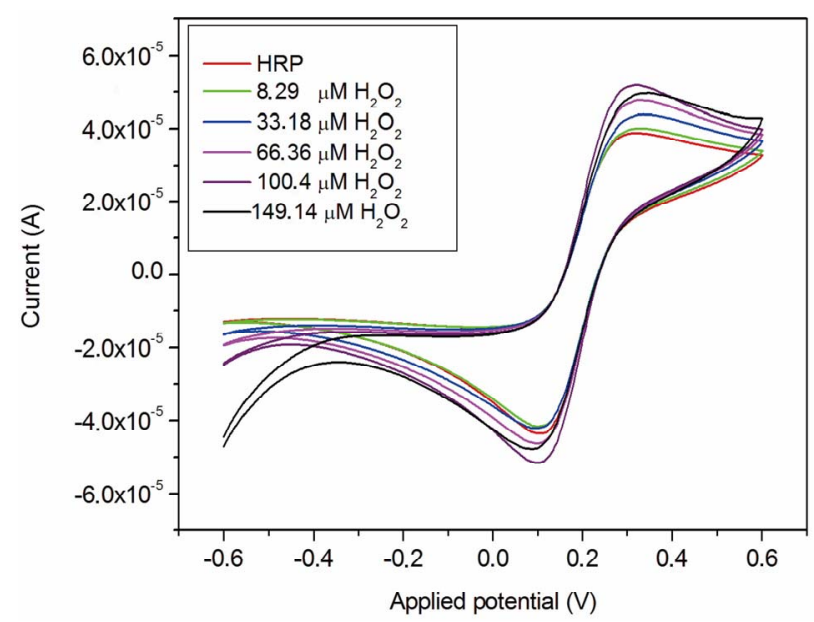

Figure 7. Cyclic voltammogram of the gold electrode functionalized with HRP enzyme before and after $\mathrm{H}_{2} \mathrm{O}_{2}$ injection. 
Figure 8 shows the impedance spectra of the gold electrode functionalized with HRP enzyme before and after injection of $\mathrm{H}_{2} \mathrm{O}_{2}$. The charge transfer resistance decrease due to current increases with increasing $\mathrm{H}_{2} \mathrm{O}_{2}$ concentration which agrees with result shown in Figure 7.

The addition of the hydrogen peroxide to the buffer solution increases the steady state current. Figure 9 shows the calibration curves of the biosensor exposed to differrent concentration of hydrogen peroxide obtained from Figure 7 (at a potential of $-200 \mathrm{mV}$ ).

The curves were represented by a linear regression and a best sensitivity was obtained. A limit detection of $4 \mu \mathrm{M}$ of $\mathrm{H}_{2} \mathrm{O}_{2}$ was obtained with a good reproductibility for the developed biosensor. This limit detection is better than the previous obtained results [16]. The biosensor response depends on the conductivity and the large surfaceto-volume ratio attained with multiwall carbon nanotubes.

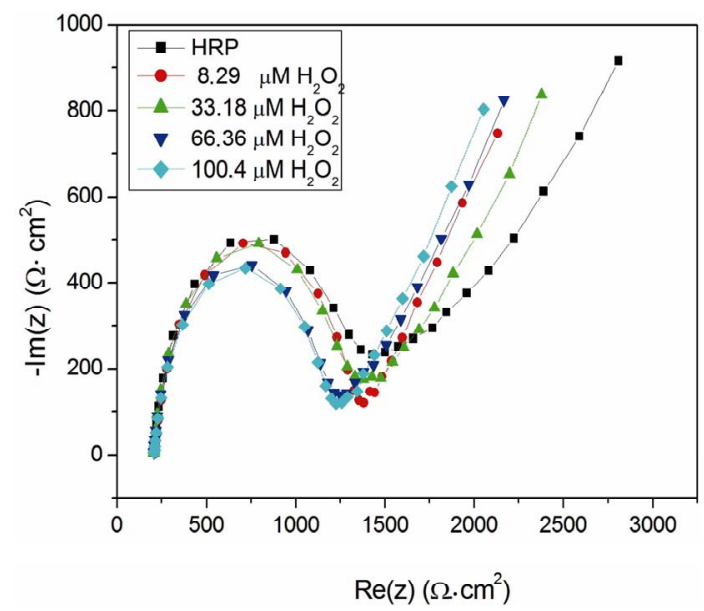

Figure 8. Impedance spectra of the gold electrode functionalized with HRP enzyme before and after $\mathrm{H}_{2} \mathrm{O}_{2}$ injection.

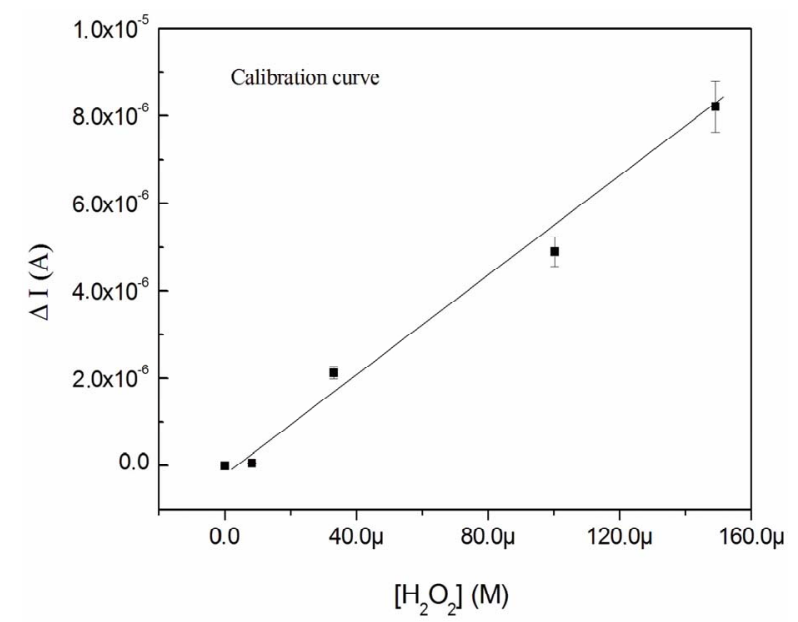

Figure 9. Calibration curve of the biosensor exposed to different concentration of hydrogen peroxide.

\section{Conclusion}

In this work, we used gold labeled multiwall carbons nanotubes for peroxidase biosensor. The labeled carbon nanotubes can be immobilized on gold electrode with the Airbrushing technique. The stability and the molecular structure of the labeled multiwall carbon nanotubes were characterized with cyclic voltammetry, impedance spectroscopy and Fourrier Transform Infra-Red spectroscopy (FTIR). For streptavidin-HRP immobilization, the labeled gold nanotubes were activated with thiol-acid and with EDC/NHS. The developed biosensor can detect $\mathrm{H}_{2} \mathrm{O}_{2}$ concentration in the range $4 \mu \mathrm{M}-160 \mu \mathrm{M}$. The results show that the biosensor response depends on the conductivity and the large surface-to-volume ratio attained with multiwall carbon nanotubes. The response of the developed biosensors was reproducible and with higher stability.

\section{REFERENCES}

[1] C. N. Rao, B. C. Satishkumar, A. Govindaraj and M. Nath, "Nanotubes," ChemPhysChem, Vol. 2, No. 2, 2001, pp. 79-105.

[2] J. Wang, "Carbon-Nanotube Based Electrochemical Biosensors," Electroanalysis, Vol. 17, No. 1, 2005, pp. 7-14. doi:10.1002/elan.200403113

[3] R. H. Baughman, A. Zakhidov and W. A. de Heer, "Carbon Nanotubes: The Route toward Applications," Science, Vol. 297, No. 5582, 2002, pp. 787-792. doi:10.1126/science. 1060928

[4] A. Starr, V. Joshi, S. Skarupo and D. Thomas, "Gas Sensor Array Based on Metal-Decorated Carbon Nanotubes," Journal Physical Chemistry B, Vol. 110, No. 42, 2006, pp. 21014-21020. doi:10.1021/jp064371z

[5] I. Jimeneza, A. Cirera, A. Cornet, J. R. Morante and I. Garcia, "Pulverisation Method for Active Layer Coating on Microsystems," Sensors and Actuators B, Vol. 84, No. 1, 2002, pp. 78-82.

[6] R. Legrib, "Design, Fabrication and Characterization of Gas Sensors Based on Nanohybrid Materials," Ph.D. Thesis, Universitat Rovira i Virgili, Tarragona, 2010,

[7] A. Abdelghani, S. Hleli and K. Cherif, "Optical and Electrochemical Characterization of Self-Assembled Octadecyltrichlorosilane Monolayer on Modified Silicon Electrode," Material Letters, Vol. 56, No. 6, 2002, pp. 1064 1068. doi:10.1016/S0167-577X(02)00678-X

[8] Y. Hou, S. Helali, A. Zhang, N. Jaffrezic-Renault, C. Martelet, J. Minic, T. Gorojankina, M.-A. Persuy, E. PajotAugy, R. Salesse, F. Bessueille, J. Samitier, A. Errachid, V. Akimov, L. Reggiani, C. Pennetta and E. Alfinito, "Immobilization of Rhodopsin on a Self-Assembled Multilayer and Its Specific Detection by Electrochemical Impedance Spectroscopy," Biosensors and Bioelectronics, Vol. 21, No. 7, 2006, pp. 1393-1402. doi:10.1016/j.bios.2005.06.002

[9] S. Hleli, A. Abdelghani and A. Tlili, "Impedance Spectros- 
copy Technique for DNA Hybridization," Sensors, Vol. 3, No. 10, 2003, pp. 472-479. doi:10.3390/s31000472

[10] S. Hleli, C. Martelet, A. Abdelghani, N. Burais and N. Jaffrezic-Renault, "Atrazine Analysis Using an Impedimetric Immunosensor Based on Mixed Biotinylated Self-Assembled Monolayer," Sensors and Actuators B, Vol. 113, No. 2, 2006, pp. 711-717. doi:10.1016/j.snb.2005.07.023

[11] J. R. MacDonald, “Impedance Spectroscopy,” Wiley, New York, 1987.

[12] H. Hillebrandt A. Abdelghani, C. Abdelghani and E. Sackmann, "Electrical and Optical Characterization of Thrombin-Induced Permeability of Cultured Endothelial Cell Monolayers on Semiconductor Electrode Arrays," Applied Physics A, Vol. 73, No. 5, 2001, pp. 539-546. doi: $10.1007 / \mathrm{s} 003390100879$

[13] W. Gerlald, "Fundamental Principles of the Electric Properties of Supported Lipid Membranes Investigated by Ad- vanced Methods of Impedance Spectroscopy,” Ph.D. Thesis, Technishe Universität of München, Munich, 1999.

[14] I. Hafaid, S. Chebil, H. Korri-Youssoufi, F. Bessueille, A. Errachid, Z. Sassi, Z. Ali, A. Abdelghani and N. JaffrezicRenault, "Effect of Electrical Conditions on an Impedimetric Immunosensor Based on a Modified Conducting Polypyrrole," Sensors and Actuators B, Vol. 144, No. 1, 2010, pp. 323- 331. doi:10.1016/j.snb.2009.08.058

[15] A. Tlili, A. Abdelghani, S. Hleli and M. A. Maaref, "Electrical Characterization of a Thiol SAM on Gold as a First Step of the Fabrication of an Immunosensors Based on a Quartz Crystal Microbalance," Sensors, Vol. 4, 2004, pp. 105-114.

[16] H. Baccar, T. Ktari and A. Abdelghani, "Functionalized Palladium Nanoparticles for Hydrogen Peroxide Biosensor," International Journal of Electrochemistry, 2011, Article ID: 603257. 